\title{
National Security Science and Technology Initiative: Air Cargo Screening
}

\author{
Philip Bingham \\ Oak Ridge National Laboratory \\ John Bush \\ Battelle Memorial Institute \\ Biays Bowerman \\ Brookhaven National Laboratory \\ Ernesto Cespedes \\ Idaho National Laboratory \\ Timothy White \\ Pacific Northwest National Laboratory
}

Prepared by Oak Ridge National Laboratory, Oak Ridge, TN 37831 managed by UT-Battelle, LLC for the U.S. Department of Energy under contract DE-AC05-00OR2225

PNNL Document: PNNL-19764

ORNL Document: ORNL/TM-2010/298

Approved for Public Release 


\subsection{Background}

The non-intrusive inspection (NII) of consolidated air cargo carried on commercial passenger aircraft continues to be a technically challenging, high-priority requirement of the Department of Homeland Security's Science and Technology Directorate (DHS S\&T), the Transportation Security Agency and the Federal Aviation Administration. The goal of deploying a screening system that can reliably and costeffectively detect explosive threats in consolidated cargo without adversely affecting the flow of commerce will require significant technical advances that will take years to develop. To address this critical National Security need, the Battelle Memorial Institute (Battelle), under a Cooperative Research and Development Agreement (CRADA) with four of its associated US Department of Energy (DOE) National Laboratories (Oak Ridge, Pacific Northwest, Idaho, and Brookhaven), conducted a research and development initiative focused on identifying, evaluating, and integrating technologies for screening consolidated air cargo for the presence of explosive threats. Battelle invested $\$ 8.5 \mathrm{M}$ of internal research and development funds during fiscal years 2007 through 2009. The primary results of this effort are described in this document and can be summarized as follows:

- Completed a gap analysis that identified threat signatures and observables, candidate technologies for detection, their current state of development, and provided recommendations for improvements to meet air cargo screening requirements.

- Defined a Commodity/Threat/Detection matrix that focuses modeling and experimental efforts, identifies technology gaps and game-changing opportunities, and provides a means of summarizing current and emerging capabilities.

- Defined key properties (e.g., elemental composition, average density, effective atomic weight) for basic commodity and explosive benchmarks, developed virtual models of the physical distributions (pallets) of three commodity types and three explosive benchmarks combinations, and conducted modeling and simulation studies to begin populating the matrix of commodities, threats, and detection technologies.

- Designed and fabricated basic (homogenous) commodity test pallets and fabricated inert stimulants to support experiments and to validate modeling/simulation results.

- Developed/expanded the team's capabilities to conduct full-scale imaging (neutron and xray) experiments of air cargo commodities and explosive benchmarks.

- Conducted experiments to improve the collection of trace particles of explosives from a variety of surfaces representative of air cargo materials by means of mechanical (air/vibration/pressure), thermal, and electrostatic methods.

\subsection{Return on Investment}

Return on investment for a project that spans basic research through systems engineering/manufacturing, and includes Battelle and four national laboratories is difficult to quantify. In the following subsection, return on investment is described for areas that can be measured (funded efforts, publications, equipment, invention disclosures, employees, contacts, and proposals); however, this project has significant returns that are difficult to measure. These non-quantifiable returns on Battelle's investment include:

1. Working relationships between the labs and Battelle - The team of researchers and their respective business offices have worked well together throughout the initiative and have 
developed an understanding and respect for capabilities of the team members and their organizations which will likely lead to joint future efforts in this application and others.

2. Air cargo application knowledge gained - Air cargo inspection is a fairly new research space because, until recently, there had been limited market or legislated demand for specific technology solutions. As a result, the research team that was brought together had an extensive expertise and capabilities on a number of potential inspection technologies, but only limited knowledge of the air cargo application. The knowledge gained on commodity types, threat types, operational requirements, limitations of existing inspection systems, etc. will enable these researchers to pursue other calls for research proposals in this area, particularly when the funding agencies move past the short term deadlines and the certified shipper program to longer term inspection modality development.

3. Reputation and relationships built with potential funding agencies - Major efforts were put forth in the final year of the project to secure funding from potential sponsors. The relationships built, in particular with the DHS S\&T Explosives Division, will result in future funded projects.

4. Multi-agency CRADA expertise - The business development and legal staff at Battelle and the labs gained valuable experience in developing and implementing, and executing a complex Cooperative Research and Development Agreement. This CRADA experience will be invaluable in establishing future multi-lab collaborations.

\subsubsection{Funded Efforts}

1. "Characterization of Adhesive properties of Homemade and Military Explosives and Evaluation of Innovative Approaches for Enhanced Explosives Particle Removal/Harvesting," conducted by Idaho National Laboratory (INL) for Directorate of Science and Technology, U.S. Department of Homeland Security, Explosives Division (\$347K in funding)

2. "Air-Cargo Vapor-Detection Support", conducted by PNNL for the Technical Support Working Group (TSWG), \$297K in FY10

3. "Associated Particle Tagging in Magnetic Spectrometers," conducted by PNNL for NA-22, \$300 K in FY10.

4. "Advanced Nondestructive Assay for Safeguards," PNNL Sustainable Nuclear Power Initiative (SNPI) LDRD, \$450k in FY10, expect \$400 K in FY11, \$350 K in FY12.

5. "High-Energy Delayed Gamma-Ray Spectroscopy for Direct Measurement of Pu in Spent Fuel," conducted by PNNL for NNSA NA-24 Next Generation Safeguards Initiative, \$150 K in FY10, expect $\$ 250 \mathrm{~K}$ in FY11

6. "Spectroscopic X-ray Computed Tomography for Improved Explosives Detection," PNNL Initiative for Explosives Detection (LDRD), \$50 K in FY09, \$180 K in FY10

7. "Lead Slowing-Down Spectroscopy for the Direct Measurement of Pu in Spent Fuel," conducted by PNNL for DOE Nuclear Energy Materials Protection Control and Accountability for Transmutation Program, $\$ 350 \mathrm{~K}$ in FY10, expect $\$ 350 \mathrm{~K}$ in FY11.

8. "Evaluation of Lead Slowing-Down Spectroscopy for the Direct Measurement of Pu in Spent Fuel," conducted by PNNL for NNSA NA-24 Next Generation Safeguards Initiative, \$75 K in FY10, expect $\$ 75 \mathrm{~K}$ in FY11

9. "Short Lived Isotopes Project," conducted by PNNL for Nonproliferation Research \& Development (NA-22).

10. "High Energy X-ray Inspection of Munitions," conducted by INL for the U.S. Army, expect \$150K in FY11 
11. "Characterization of Nuclear Grade Graphite," conducted by INL for DOE NE, expect $\$ 50 \mathrm{~K}$ in FY11

\subsubsection{Publications}

1. Runkle, R., White, T., Miller, E., Caggiano, J., and Collins, B., "Photon and neutron interrogation techniques for chemical explosives detection in air cargo: A critical review," Nuclear Instrumentation and Methods A, 2009.

2. J.M. Beaver, R.A. Kerekes, and J.N. Treadwell. "Decision-level Information Fusion to Assess Threat Likelihood in Shipped Containers." Proceedings of the 2009 IEEE Conference on Technologies for Homeland Security, Waltham, MA, May 2009.

3. "Analysis of ionizing-radiation techniques for air cargo screening," Presented at DHS CIED laboratory days by Tim White.

4. "Evidence-based data fusion for detecting explosives in air cargo," Presented at DHS CIED laboratory days by David Gerts.

5. A.J. Edwards and E.H. Seabury, "Neutron/Gamma Spectroscopy and Transmission Experiments Through Varying Electrical Equipment Commodity Thicknesses." INL External Report Number 09-15367, January 2009.

6. T. White, "AIT [Advanced Imaging Technology] Technology Overview," invited presentation at the Algorithms Development for Security Applications Workshop, April 27-28, 2010.

\subsubsection{Equipment \& Software}

BNL

BNL purchased computers and nuclear electronics (NIM and VME) modules to support advanced modeling efforts as part of a parallel processing cluster. The electronics modules are used as components in advanced detection systems. For the inelastic scatter measurements, electronics were assembled and software developed for the BNL gamma spectroscopy system to correlate the associated particle information provided by an associated particle neutron source with the gamma signals from $\mathrm{n}$ gamma reactions. This correlated signal is a big step to reducing the high background levels usually associated with fast neutron inelastic scattering.

\begin{tabular}{|l|l|l|}
\hline Item & Purpose & Cost \\
\hline Computer cluster & Radiography modeling & $\$ 40 \mathrm{~K}$ \\
\hline Electronics & Inelastic-scatter experiments at ORNL & $\$ 18 \mathrm{~K}$ \\
\hline
\end{tabular}

INL

In support of the $x$-ray imaging tasks of the initiative, INL procured a $450 \mathrm{kVp} x$-ray generator from Yxlon, Inc and a linear detector array from Detection Technology (DT), Inc. These two components were integrated with existing INL imaging equipment (linear and rotate stages, motors and controllers, computers, data acquisition and analysis software, additional $\mathrm{x}$-ray detectors) and a $4 \mathrm{MeV}$ linac from Idaho Accelerator Works to form the horizontally translating, two-dimensional projection radiography cargo inspection prototype (Figure 1). This system provided hundreds of images of test objects (step wedges), surrogate threats and air cargo material that were subsequently used in several subtasks including multi-view imaging, multi-particle imaging, data fusion, attenuation and contrast studies. In 
the final year of the project, an enhanced imaging system was envisioned, designed and constructed. This system was designed to be fully adjustable in several configurations including source-object and source-detector distances, detector and collimated-source orientations allowing both horizontal and vertical scanning tomography, and interchangeability of $450 \mathrm{kVp}$ generator and linac. Project funds were used to procure the source and detector towers. Completion of the upgrades extended beyond the end of the project's funding cycle but the system is now operational. The system has been used for a proof of principle task for the US Army that has led to follow-on funding. Additional projects for the Department of Energy Office of Nuclear Energy are anticipated. It will also be available for any future air cargo inspection efforts that may arise.

\begin{tabular}{|l|l|l|}
\hline Item & Purpose & Cost \\
\hline x-ray generator (450kVp) & Radiography experiments & $\$ 110 \mathrm{~K}$ \\
\hline Linear detector array & Radiography experiments & $\$ 150 \mathrm{~K}$ \\
\hline Towers & Upgrades to lab-prototype imaging equipment & $\$ 40 \mathrm{~K}$ \\
\hline
\end{tabular}

ORNL

ORNL had already developed Associated Particle Neutron Radiography (APNR) system for interrogation of smaller objects (drums) prior to the start of this project. This initiative developed a new framework for the system and purchased more detectors to develop a new system capable of scanning pallet sized objects as seen in Figure 1.

For the multi-view effort, an iterative tomographic slice reconstruction code was modified to allow reconstruction with few views and no restrictions on angular positions of the views. This code will have use in applications outside of air cargo as well.

\begin{tabular}{|l|l|l|}
\hline Item & Purpose & Cost \\
\hline Imaging detectors & nAPI-radiography experiments & $\$ 15 \mathrm{~K}$ \\
\hline Support arm & Upgrade to nAPI-radiography equipment & $\$ 18 \mathrm{~K}$ \\
\hline NaI(TI) detector & Inelastic-scatter measurements & $\$ 14 \mathrm{~K}$ \\
\hline
\end{tabular}

PNNL

Active Interrogation Capability: Equipment was acquired with initiative funding in FY08 to establish the air-cargo scientific platform to advance research applications of gamma/neutron unified systems (ASPARAGUS) capability at PNNL. Equipment that was procured included neutron generators (a D-D and a D-T capability), an array of plastic scintillators and electronics, liquid scintillators, control/spectroscopy software, and a physical platform to be used for the integration of neutron transmission, inelastic scatter, elastic scatter, and thermal-capture experiments. An existing interrogation cell was identified for the location of this equipment, and PNNL contributed to preparation and instrumentation of the cell for safe operation. General Research Equipment (GRE) funding at PNNL was obtained to develop an $x-$ ray interrogation capability in the same space. Many projects at PNNL have leveraged these activeinterrogation resources.

\begin{tabular}{|l|l|l|}
\hline Item & Purpose & Cost \\
\hline $\begin{array}{l}\text { Neutron generators (2 DT } \\
\text { heads, 1 DD head) }\end{array}$ & Correlated-particle (ASPARAGUS) experiments & $\$ 201 \mathrm{~K}$ \\
\hline Imaging detectors & Neutron-radiography experiments & $\$ 35 \mathrm{~K}$ \\
\hline Liquid scintillators & Neutron-scatter experiments & $\$ 15 \mathrm{~K}$ \\
\hline
\end{tabular}




\begin{tabular}{|l|l|l|}
\hline Item & Purpose & Cost \\
\hline Computer systems & modeling, data acquisition & $\$ 17 \mathrm{~K}$ \\
\hline PNNL General Research Equipment leveraging the CRADA investment & \\
\hline Advanced Radiographic Research System (FY-09) & $\$ 500 \mathrm{~K}$ \\
\hline High-purity lead and instrumentation for construction of LSDS (FY-10) & $\$ 320 \mathrm{~K}$ \\
\hline
\end{tabular}

$\mathrm{BCO}$

Battelle Columbus Operations acquired one Arena seat license. Arena is the leading factory floor simulation software package within the US marketplace. Arena is taught in approximately 900 university systems engineering schools. ORNL, led by Dr. Rekha Pillai, has established expertise with this software tool; including with the TSA client. BCO, in its capacity as systems engineering lead on the project, acquired the license and basic training anticipating collaborative work with ORNL on alternative air cargo solutions. One Arena seat license is approximately $\$ 25 \mathrm{~K}$.
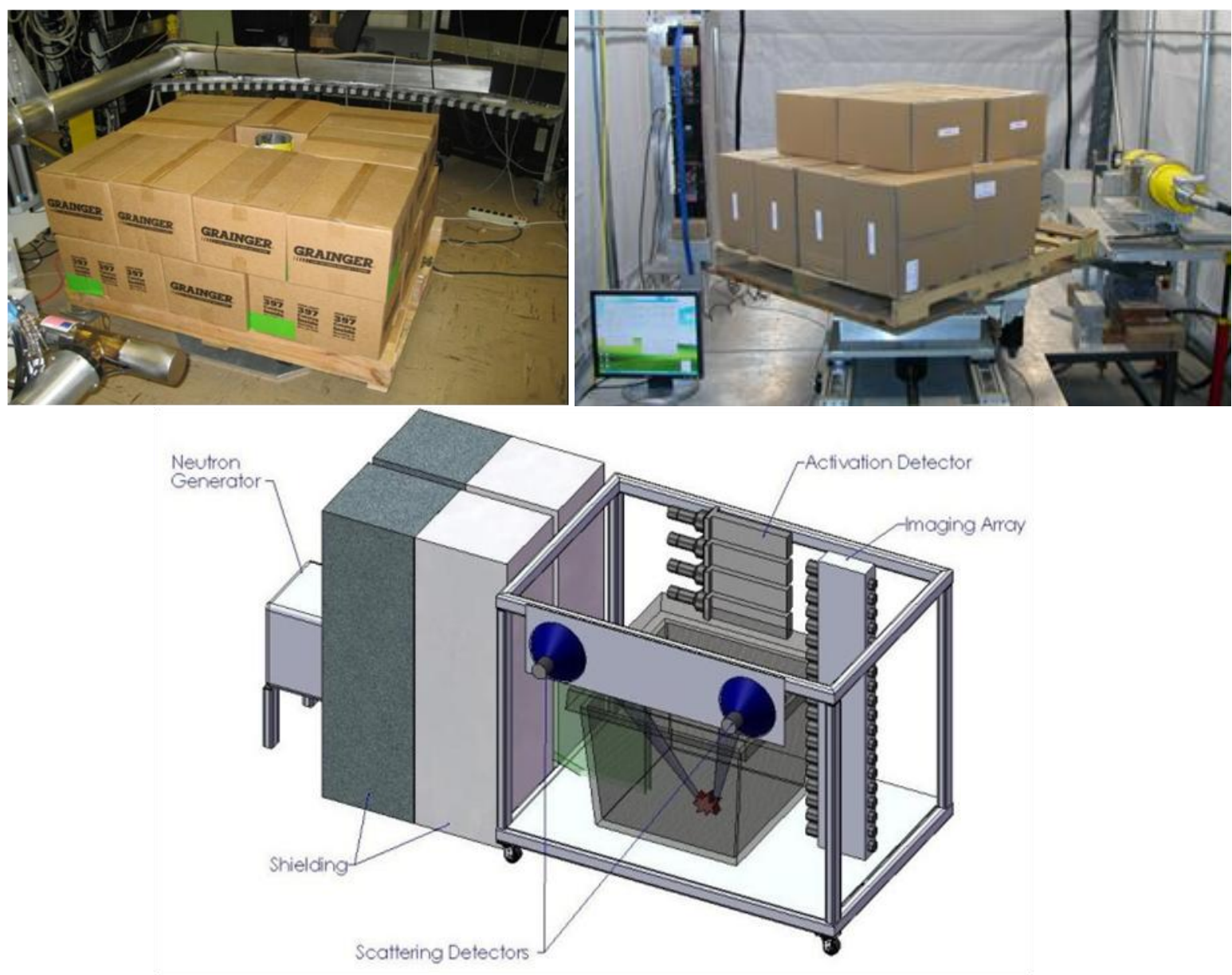

Figure 1. CRADA-purchased equipment. (top left) ORNL - associated particle neutron radiography system for pallets. (top right) INL - pallet x-ray radiography/CT system. (bottom) PNNL - correlated particle laboratory measurement system.

\subsubsection{Invention Disclosures}

1. Dual neutron/photon generator for active interrogation, Jac Caggiano, Tim White, Mitch Woodring (PNNL), IDR/IPID 16379-E 
2. Correlation of inspection data to verify contents of assembled shipping container- Bingham and Rammachandran (ORNL) IDR 200902301

3. Detection system for high-resolution gamma spectroscopy combined with time of flight filtering - Diozegi, Salwen, and Vanier (BNL)

4. Determining Basic Probability Numbers for Data Fusion - Gertz (INL)

5. Method for Explosive Detection - Edwards (INL)

6. Multiple source API for simultaneous capture of multiple projections- Bingham, McConchie, Mullens, Mihalczo, and Hausladen (ORNL) IDR 200902302

7. Multi-Particle Inspection Using Associate Particle Sources- Bingham, Hausladen, McConchie, Mullens, and Mihalczo (ORNL) IDR 201002418

8. Random Pulsed Particle Interrogator, Jac Caggiano (PNNL), IDR/PID, 16044-E

9. Gamma Imaging Improvements - Bingham and Mullens (ORNL) IDR 200802161

\subsubsection{Employees}

Eric Sword - ORNL hired Eric as a post doc. Eric initially worked on the project as a graduate student from Purdue due to our contract to use a neutron source from Purdue. His PhD research was in the field of explosives detection.

Janak Rammachandran - ORNL brought Janak into the Image Science and Machine Vision (ISMV) group in part to work on detection of threats in multi-view imagery for the cargo initiative.

Ben McDonald (PNNL) - emission computed tomography simulation, instrumentation, and analysis. Ben complements and expands our active-interrogation capability.

\subsubsection{Contacts}

1. Dr. Doug Bauer, DHS S\&T, Explosives Division - contacted ORNL with respect to associated particle neutron radiography.

2. Allan Collier, TSA Air Cargo TSNM, Branch Chief, Technology - Visited ORNL mid-December 2009. Visit included opportunity to brief him on the Battelle Air Cargo Initiative.

3. Carl Crawford, CSupTwo / DHS S\&T Explosives Division - Carl has involved PNNL, INL and ORNL in the Algorithm Development for Security Applications workshop series hosted by the ALERT Center of Excellence

4. Joseph Callerame, American Science and Engineering - the air-cargo initiative has helped PNNL foster a collaborative relationship with AS\&E on R\&D opportunities for explosives detection.

5. Dan Strellis and Luis Arroyo, Rapiscan - Visit to ORNL to discuss collaboration opportunities for filling out matrix and other areas of mutual interest.

\subsubsection{Proposals \& White Papers}

White papers submitted to DHS S\&T Air Cargo FY2010 RFI

1. High Volume Trace Explosives Sampling System for Containerized and Palletized Air Cargo, Ernesto Cespedes (INL)

2. Associated Particle Neutron Radiography for Pallet Inspection Philip Bingham, Paul Hausladen, Seth McConchie, Jim Mullens, and John Mihalczo (ORNL) Tim Roney (INL)

3. Detection and Identification of Improvised Explosive Devices at the Surface of Low, Medium, and High-density Cargo Using Neutron Induced Gamma-ray Spectroscopy, Seth McConchie, Eric Sword, John Mihalczo, Philip Bingham, and James Mullens (ORNL) David Koltick (Purdue) 
4. Detection and Identification of Improvised Explosive Devices Embedded in Low, Medium, and High-density Cargo Using Neutron Radiography and Neutron Induced Gamma-ray Spectroscopy, Seth McConchie, Eric Sword, John Mihalczo, Philip Bingham, and James Mullens (ORNL) Istvan Dioszegi, Cynthia Salwen, and Leon Forman (BNL)

5. Verification of Trusted Shipper Pallets, Philip Bingham (ORNL)

6. Whitepaper on Sensor Fusion for Air Cargo Inspection, David Gertz and Tim Roney (INL)

7. Air Cargo Scientific Platform to Advance Research Applications of Gamma/Neutron Unified Systems (ASPARAGUS), Mitchell Woodring, Timothy White (PNNL)

Proposals to the Proliferation Detection Program at NA-22, FY10

1. SNM Tracking Using A Correlated Fast Neutron and Gamma-ray Imaging Detector, J. Ely, M. Woodring, M. Myjak, T. White (PNNL)

2. Correlated Particle Measurement, Technology, and Gaps Evaluation, Tim White, James Ely, Mitch Woodring (PNNL)

3. Time Correlated Fast Neutron and Gamma-ray Localization of SNM, J. Ely, M. Woodring, M. Myjak, T. White, S. Robinson (PNNL)

White papers submitted (or to be submitted) to DHS S\&T FY2010 Long Range BAA

1. Associated Particle Neutron Radiography for Pallet Inspection

Philip Bingham, Paul Hausladen, Seth McConchie, Jim Mullens, and John Mihalczo (ORNL) Tim Roney (INL) - Selected for full proposal to be submitted Aug 2010.

Full Proposal submitted to DHS S\&T FY2010 Long Range BAA

1. Associated Particle Neutron Radiography for Pallet Inspection Philip Bingham, Paul Hausladen, Seth McConchie, Jim Mullens, and John Mihalczo (ORNL) Tim Roney (INL) - Reviewed and not selected for funding. Reasons for not funding were concerns over cost and time to product.

Full Proposal submitted to DHS S\&T BAA 08-01

1. Air Cargo Screening: A Battelle/Multi-Lab Initiative for Explosive Threat Detection in Consolidated Air Cargo, Ernesto Cespedes (INL), Michael Fisher (Battelle). 


\section{Technical Summary}

Technically, the air cargo initiative went through a major directional change within the first year as the effort shifted from a short-term, tool-development project into a research project to investigate potential inspection methods for cargo. The following subsections provide overall descriptions of the efforts in each of the two years and point to appendices for each major thrust area that contain technical details and results from the effort.

\section{$2.1 \quad$ Fiscal Year 2007-2008}

The air cargo initiative began with the goal of developing an air cargo inspection system in response to the congressional requirement for $100 \%$ cargo scanning by August 2010 . In the first year of funding, the team was split into two groups. The first group was to concentrate on engineering issues for system development and the second group was to identify inspection methods to be incorporated into the system. Midway through the first year, the team determined that there was not a market for a scanning tool of this type in the short term, as TSA was moving toward a certified-shipper program to meet the screening requirements. At this point, the emphasis shifted to research of methods for threat detection with the notion that inspection systems will eventually be needed, but on a longer time scale. A broad range of research areas were addressed in the first year of the initiative, with many of these leading into the thrust areas of the second year. Three of the broad research areas in the first year were building blocks for the activities in the second year and possible follow-on activities. These activities included a gaps analysis, initial development of commodity benchmarks and threat simulants, and modification/development of laboratory based systems ( $x$-ray, neutron, and correlated emissions) for air cargo pallets. These three areas are detailed in the following sub-sections. The research effort for FY08 was organized through a set of research plans. For a more detailed view of all the topics covered and presented results for FY08, see the research plan descriptions ( $\backslash$ Research Plans $\backslash^{*}$.doc) and the end of FY reporting performed for each of these areas (\Meetings $\backslash 2008$ Aug 26-28 POC meeting at ORNL\Presentation Files $\backslash *$.ppt).

\subsubsection{Gaps analysis}

A gaps analysis was performed with respect to detection of Explosive/Radiological/Nuclear (ERN) threats. Figure 2 provides a breakdown of signatures that can be used to detect and/or identify these threats along with candidate technologies for each of the signatures. 


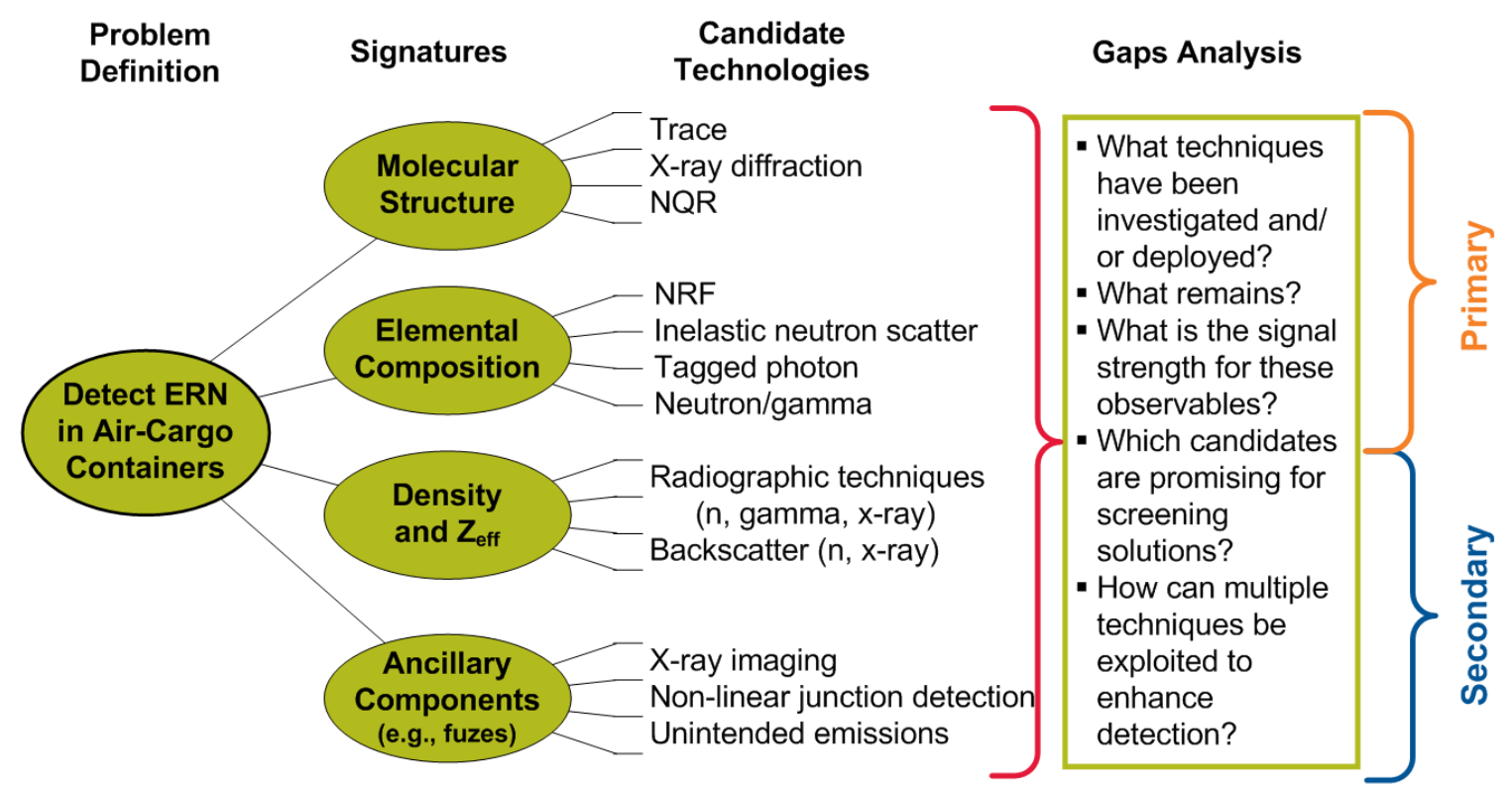

Figure 2. Organization of candidate detection technologies.

Using this approach, a key result from the analysis that was performed is that no single detection method is capable of detecting all threats in all air cargo commodity combinations. This result led to the second key result which is the development of a concept for air cargo flow through inspection with multiple stages such that an initial high speed inspection may be used to determine if there are any anomalies. If no anomalies are detected, the cargo is passed on for shipping. If an anomaly is found, a secondary inspection is performed using technologies that more precisely interrogate the area of concern and determine whether the anomaly is a threat. A price that is paid for these secondary inspections is longer interrogation times; diverting a vast majority of (benign) cargo in a rapid primary step will abate throughput concerns. In Figure 3, a graphical depiction of this concept is shown with candidate technologies placed in positions within the flow based on their impact on the flow of cargo and their ability to identify an object as a threat.

The third key accomplishment of the gaps analysis was a definition/organization of the air cargo threat detection space. Figure 4 shows a simple graphic for a three-dimensional organization of the detection space with threats on one axis, commodities on the second axis, and potential detection technologies on the third axis. With this concept, a matrix was developed as seen in Figure 5 to organize the assessment of technologies for each threat/commodity combination. In this figure, a twodimensional matrix is shown; a single box in this table would represent the ability of a single technology to detect a certain threat in that commodity type. A similar table can be developed for each candidate technology, resulting in a three-dimensional matrix that indicates which technologies have the highest chance of detecting a particular threat in a particular commodity type.

The final accomplishment of the gaps analysis was the publication of "Photon and neutron interrogation techniques for chemical explosives detection in air cargo: A critical review" in the journal Nuclear Instrumentation and Methods A. 


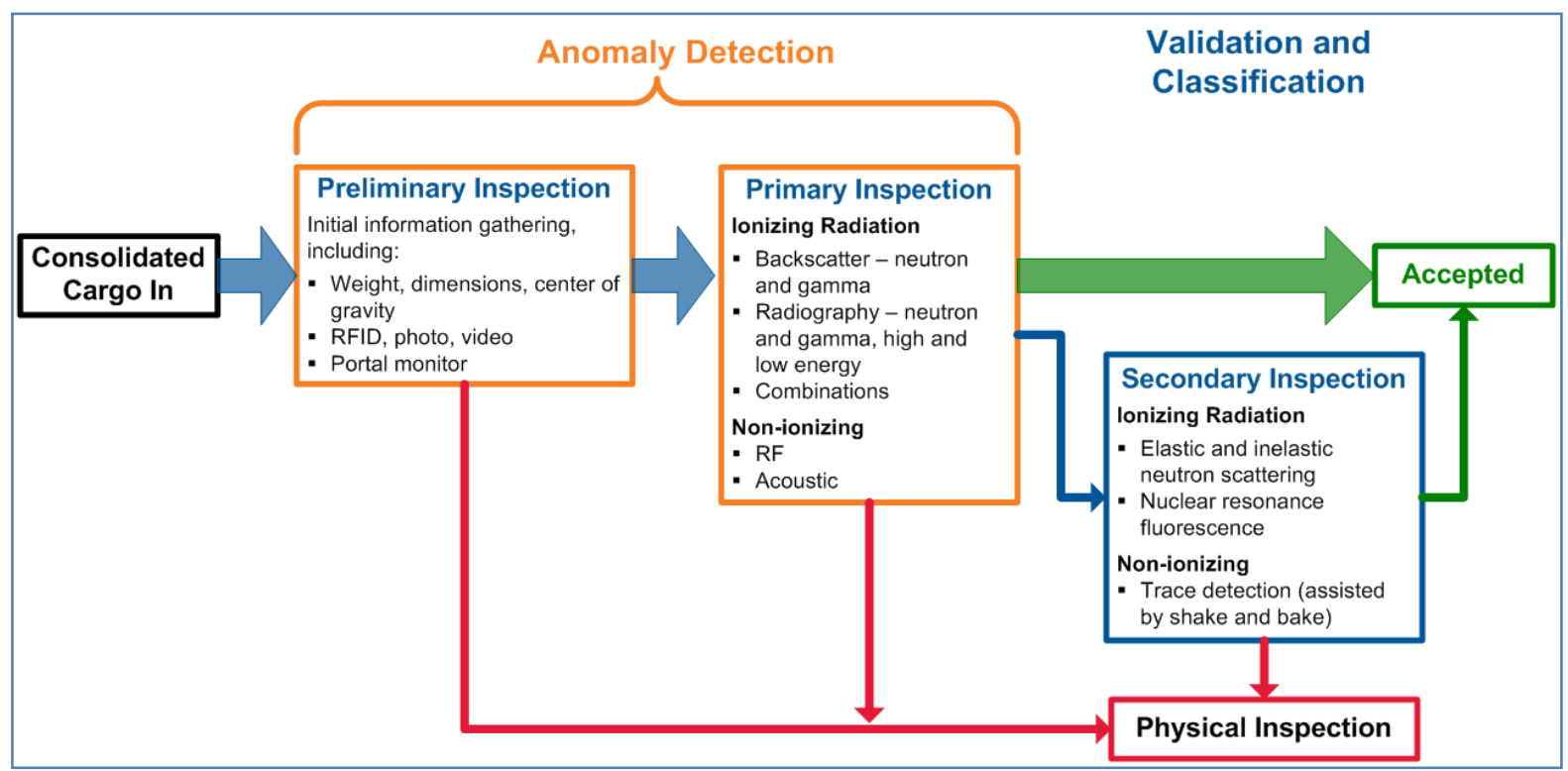

Figure 3. Design of cargo inspection flow with respect to candidate technologies.

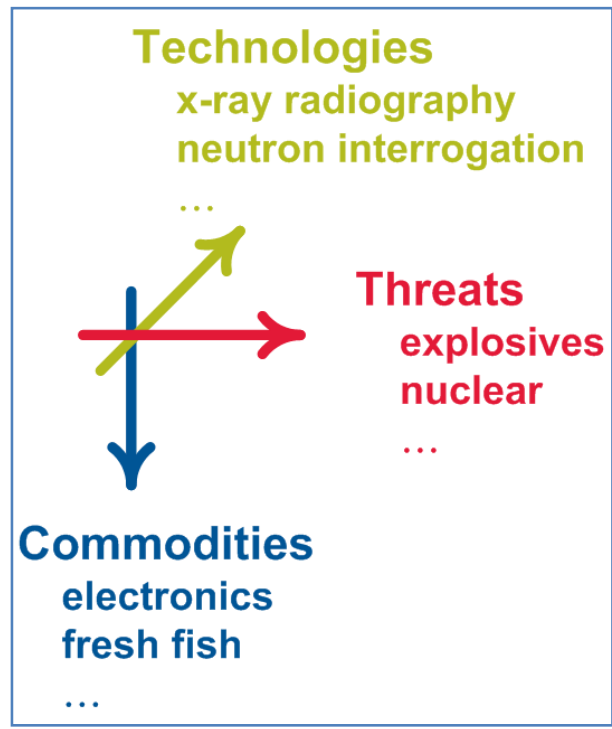

Figure 4. Definition of air cargo threat detection space. 


\begin{tabular}{|c|c|c|c|c|c|c|c|c|c|c|c|c|}
\hline & & \multicolumn{3}{|c|}{ Explosive Benchmarks } & \multicolumn{3}{|c|}{ Nuclear Benchmarks } & \multicolumn{3}{|c|}{ Radiological Benchmarks } & \multicolumn{2}{|c|}{$\begin{array}{c}\text { Security } \\
\text { Benchmarks }\end{array}$} \\
\hline & & $\begin{array}{c}\text { C-4 } \\
\text { (brick) }\end{array}$ & $\begin{array}{l}\text { Tovex } \\
\text { Extra } \\
\text { (tube) }\end{array}$ & $\begin{array}{l}\text { PETN } \\
\text { (sheet) }\end{array}$ & $\begin{array}{c}\text { DU } \\
\text { sphere in } \\
\text { PB \& } \\
\text { Lexan } \\
\text { cube }\end{array}$ & $\begin{array}{c}\text { DU } \\
\text { cylinder } \\
\text { infee } \\
\text { cylinder }\end{array}$ & $\begin{array}{c}\text { DU } \\
\text { spherical } \\
\text { shell } \\
\text { embedded } \\
\text { in Lexan }\end{array}$ & $\begin{array}{l}\text { HEU } \\
\text { sphere } \\
\text { in Fe } \\
\text { shell }\end{array}$ & $\begin{array}{c}\mathrm{PU} \\
\text { sphere in } \\
\text { multi- } \\
\text { layered } \\
\text { shielding }\end{array}$ & $\begin{array}{l}\text { Cs-glass } \\
\text { sphere in } \\
\text { PB shell }\end{array}$ & $\begin{array}{c}\text { Contra- } \\
\text { band }\end{array}$ & $\begin{array}{l}\text { Stow- } \\
\text { aways }\end{array}$ \\
\hline \multirow{11}{*}{ 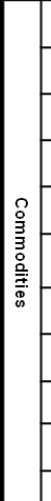 } & Electronics and Computer Equipment (EE) & & & & & & & & & & & \\
\hline & Printed Material (PM) & & & & & & & & & & & \\
\hline & FreshFlowers (FF) & & & & & & & & & & & \\
\hline & Machine Parts (MP) & & & & & & & & & & & \\
\hline & Perishables, Grains, Animal Feed (PR) & & & & & & & & & & & \\
\hline & Non-Metallic Mineral Products (MDG) & & & & & & & & & & & \\
\hline & Seafood and Shellfish (SF) & & & & & & & & & & & \\
\hline & Chemicals, Alcohol, Glass (CHEM) & & & & & & & & & & & \\
\hline & Wearing Apparel (WA) & & & & & & & & & & & \\
\hline & Paper Products (non printed) (PP) & & & & & & & & & & & \\
\hline & Multiple Commodities (MULT) & & & & & & & & & & & \\
\hline
\end{tabular}

Figure 5. Threat/commodity detection matrix for a single detection technology.

\subsubsection{Commodity benchmarks and threat simulants}

With the Gaps analysis and resulting threat/commodity matrix, a key task was to define the commodities and threats at a level that would allow the research teams to evaluate probability of detection for the candidate technologies through modeling and testing. First, a set of explosive, radiological and nuclear threats were developed. Explosive simulants that are nearly identical in chemical content and density were acquired through Battelle to allow testing with respect to elemental, density, and $Z_{\text {eff }}$ signatures. These threat and commodity definitions are incorporated in the column and row headings of the table in Figure 5.

With the wide range of commodities shipped in air cargo, obtaining a definition of typical cargo is a complicated problem that will depend on many factors including location of the airport and time of year. To define the commodity types, data was extracted from previous studies in break bulk scanning at two airports and organized according to common commodity classifications. Table 1 provides the breakdown of commodity types, the average box size, and the percentage for each commodity type from this two-airport study. Estimates of the average elemental composition and the average density for most of these commodities were developed by the team and can be found in the document Modeling Objects Definitions.xls.

Using these definitions, commodity pallet models were developed for EE, MP, PM, CHEM and WA. These models were designed to match elemental contents and density as provided by the commodity class definitions. Full pallets were fabricated for the EE, PM, and MP types and two parcel boxes for the WA type. In addition to the commodity boxes for the pallets, the design allowed for and provided inserts for the boxes to locate threat simulants at know locations within the pallets for testing. Figure 6 shows examples of the EE and MP pallets with a cutout for a threat shown between the boards in the EE box. 
Table 1. Commodity class definitions.

\begin{tabular}{|c|c|c|c|c|}
\hline $\begin{array}{c}\text { Commodity } \\
\text { Class }\end{array}$ & Commodity Description & average size box [in] & $\begin{array}{c}\text { average } \\
\text { density [ft/ } \\
{\left[\mathrm{lb}^{\wedge} 3\right]}\end{array}$ & $\begin{array}{c}\text { percentage } \\
\text { of pallets }\end{array}$ \\
\hline EE & Electronics and computer equipment & $14.0 \times 13.5 \times 8.6$ & 14.8 & 40.4 \\
\hline MULT & Combination of commodities & $21.2 \times 11.5 \times 10.8$ & 26.2 & 30.3 \\
\hline PM & Printed material & $20.5 \times 16.9 \times 14.5$ & 19.5 & 22.5 \\
\hline $\mathrm{FF}$ & Fresh flowers & $41.0 \times 15.8 \times 3.3$ & 15.2 & 11.5 \\
\hline MP & machine parts & $20.5 \times 15.7 \times 12.4$ & 13.4 & 9.3 \\
\hline PR & Perishables, grains, animal feed & $26.0 \times 24.8 \times 3.9$ & 19.1 & 8.4 \\
\hline MDG & non-metallic mineral products & $15.7 \times 9.5 \times 9.1$ & 15.8 & 7.1 \\
\hline SM & seafood and shellfish & $23.6 \times 19.7 \times 12.0$ & 15 & 5.5 \\
\hline CHEM & chemicals, alcohol, glass & $18.5 \times 12.4 \times 12.4$ & 25.5 & 2.4 \\
\hline WA & wearing apparel & $24.6 \times 15.0 \times 11.0$ & 10.4 & 1.2 \\
\hline PP & paper products (non printed) & $16.3 \times 16.2 \times 11.0$ & 10 & 0.7 \\
\hline
\end{tabular}
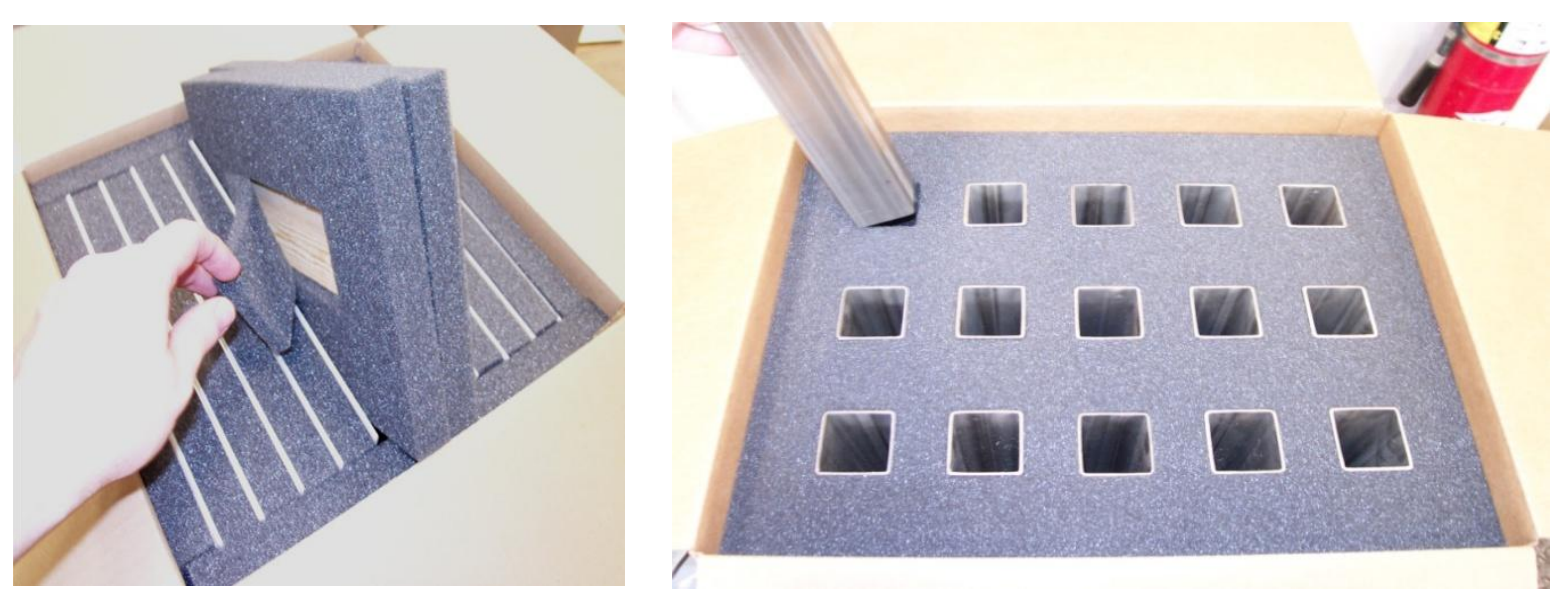

Figure 6. Physical models of heterogeneous commodities used for benchmark experiments. On the left is the EE commodity with simulated threat (partially removed from the box), and on the right is the MP commodity model.

\subsubsection{Laboratory based pallet inspection systems development}

The third key effort in the first fiscal year was the design/development of equipment to test candidate technologies on the pallet size commodity benchmarks. Three pieces of equipment were design or modified to perform these studies: $x$-ray radiography, associated particle neutron radiography, and correlated particle measurements. These three systems were introduced in the return on investment section of the report and can be seen in Figure 1. 
The FY09 effort focused on five thrust areas that were developed following the publication of the gaps analysis. The Multi-particle, Multi-energy, Multi-view Imaging Thrust concentrated on the use of radiographic imaging techniques and investigating use of various numbers of interrogation energies, particles (neutrons, gamma-rays, and $x$-rays) and views to improve detection of explosives over a range of commodity types. Imaging methods in this thrust area depend on detection of anomalies with signatures similar to explosives. The Secondary Inspection Techniques Thrust included methods for clearing anomalies and identification of potential threats. These methods would focus on an area identified by a primary detection method as suspicious. Due to the wide range of threats and commodities, no single detection method is capable of identifying explosives in air cargo. A key premise of this work is that the identification capabilities can be improved using signatures from multiple inspection methods. The Data Exploitation Thrust investigated the combinations of these signatures using a knowledge of the physics of each method along with known pattern recognition algorithms. The Non-lonizing Radiation Thrust focused on RF methods for location of active electronics within the container and on "shake and bake" methods for improved trace detection. A final thrust, HighRisk/High-Return Investigations explored technically challenging concepts with a long path to deployment, but that could make a significant impact on explosives detection or system design. Projects in this area included a photon-tagging concept and methods to use scattered radiation for improved material identification and localization. In addition to the technical thrust areas, a significant level of effort was expended by the team in planning a path forward for development of a multi-sensor air cargo screening system that includes components from all the thrust areas. This effort resulted in a 5 year project execution plan that is a part of this report package under the filename Battelle 2009 Air Cargo PEP 3-17.doc.

\subsubsection{Multiple-Particle/Energy/View Imaging Thrust}

The key area of effort for the MPEVI (Multiple-Particle/Energy/View Imaging) thrust was the study of using multiple particles and energies to improve signatures for detection of explosives. In this area, we investigated $\mathrm{x}$-ray, gamma ray, and neutron energies that provided potential for explosives detection over the range of commodities. Capabilities for imaging pallets with low $(<450 \mathrm{KeV})$ and high $(4 \mathrm{MeV})$ energy $\mathrm{x}$-rays, gamma rays (Cf-252 and Co-60), and neutrons (DT [14.1MeV] and DD [2.45MeV] generators) were developed at the partner laboratories to perform tests on threat detection. Simulation capabilities were verified against the physical tests and used to extend understanding of the potential limitations of each technique as they are applied to the different commodities. This project developed a metric initially proposed in the gaps analysis study and used it to compare $x$-ray radiography at various energies (multiple-energy research plan), and to compare x-ray radiography with neutron radiography (multiple-particle research plan). The contrast metric has been calculated for simple objects (step wedges) and for simple heterogeneous objects. A small subset of the data collected over the course of the project has been analyzed and appears in the multiple-energy, multiple-particle report (Multiple-Particle Multiple-Energy Investigation (PNNL-19492).docx). The data show that neutron radiography has the potential to improve the contrast of a photon radiograph, but the mechanism for combining the information is not clear. Two other reports from the initiative address combining the information from multiple particles: Correlated-Particle Interrogation (PNNL-19536).docx discusses the R-value approach with different sources of neutrons and x-rays or gamma rays, and Data Fusion for Air Cargo Explosive Screening v3.pdf discusses using data from two different $\mathrm{x}$-ray generators.

In the multiple view portion of the MPEVI thrust, the air cargo team has performed initial software development and investigations into the use of multiple projections through a cargo container to improve detection of explosive threats within the commodity. This task is applying the basic concepts that underlie computed tomography, but attempting to use fewer projections to reduce scan time and 
therefore have a smaller impact on throughput. The collection of few views for screening has begun to see application in screening of carry-on baggage; such systems are labeled as Advanced Technology (AT) systems by TSA. Over the course of this project, the team has performed background research into laminography, investigated the depth resolution required for detectability of explosive threats, performed initial studies of basic laminography reconstruction, and modified an iterative CT reconstruction code to allow reconstruction from sparse views. As described in the following, the depth resolution calculations are very promising with only a few depth slices required in each commodity to bring our explosive threats to a detectable contrast level. Calculations performed with an easily implemented algorithm (the "shift and add" approach) pointed to the need for an algorithm using a more complete system model, and an iterative reconstruction code has been developed and tested on phantom data to evaluate reconstruction performance with few views. These tests have shown improved resolution and accuracy for the phantom data. Two x-ray air cargo data sets have been specifically collected at INL to test performance with real data and are available for future efforts in the multi-view thrust area.

The completed effort under the multi-view task has shown that laminography approaches have the ability to provide coarse resolution in depth and that the explosive threat simulants require depth resolution for detection within our defined commodities of $E E, P M, F F, M P$, and CHEM but the resolution can be coarse $(30 \mathrm{~cm}$ thick for the Tovex and Semtex simulants, and $8 \mathrm{~cm}$ thick for the PETN sheet stimulant). The next step for the iterative reconstruction code is to separate the voxel dimensions such that the size in the direction from the source to the detector can be increased for reconstruction of a few radiographic slabs to present to the detection algorithm or human reviewer. With the iterative reconstruction code and the collected $x$-ray data, immediate follow-on efforts for air cargo would be to perform a performance analysis on the reconstructions with these data sets and continue this effort by gradually increasing the complexity of the pallet. Longer-term follow-on efforts for this technology should include use of few view reconstruction in conjunction with multiple energies or multiple particles (x-ray and neutron) to improve material detection for layers of a pallet. Outside the air cargo application, there is a potential for follow-on funding to test few view reconstruction for other applications where time or geometry limits data collection to a few views of an object. The technical report for the Multi-View thrust can be found in $\mathrm{ACl}$ - Close out document (Multi-view)(2-20-10).docx.

\subsubsection{Secondary Inspection Thrust}

The purpose of secondary inspection methods is to resolve anomalies and identify explosives. The FY09 effort in this thrust area has been divided into three areas: 1. Correlated-Particle Interrogation, 2. nAPI inelastic Scatter, and 3. Nuclear Resonance Fluorescence (NRF). The correlated-particle project was predicated on an assumption that multiple signatures are available when interrogating an object with either photons or neutrons and that techniques that observe only one of the available signatures are ignoring information that could be used for threat identification. A test platform, the air-cargo scientific platform to advance research applications of gamma/neutron unified systems (ASPARAGUS) was developed at one of the partner labs in order to test detectability of threats against the matrix of commodity items. This work is reported on in Correlated Particle Interrogation (PNNL-19536).docx.

The neutron radiography system used in the MPEVI thrust uses neutron associated particle imaging (nAPI) to improve image quality by rejecting scattered neutrons (a form of electronic collimation). In $\mathrm{nAPI}$, the initial track of the ingoing neutron is determined by the direction of an alpha particle emitted in the opposite direction. With this track and appropriate timing information, gamma-detector data can be filtered to measure primarily gamma emission resulting from inelastic scatter. Gamma energy information and neutron track information can then be interpreted to provide a 3D map of material composition. By performing this measurement with the nAPI system, a neutron transmission radiograph 
is captured simultaneously. In this project, gamma detection capability was added to the nAPI system to allow inelastic scatter measurements. Measurements were performed to verify simulations and determine explosives identification limitations. The main finding of experiments conducted in the spring was that measurements required hours of exposure to obtain signals from pure calibration standards. Higher neutron flux and additional detectors offer the hope of explosive identification in commodity materials, but additional experiments will have to be conducted under future funded projects.

The final project under the secondary inspection techniques is NRF. NRF uses a Bremsstrahlung source to interrogate the object under test. Resonant excitation of the target nucleus and subsequent relaxation results in characteristic gamma emission in the MeV energy range. Energy signatures are then used for material composition identification. This method is limited by the available interrogation time and the flux from the source. Detection limits and efficiency questions were addressed through calculations, simulations, and experiments at the Idaho Accelerator Center. This work demonstrated for the first time the detection of nitrogen lines in simulated explosive material. This method has the potential of being integrated with an $\mathrm{x}$-ray radiography system in primary to save costs and container routing overhead.

\subsubsection{Data Fusion Thrust}

From the gaps analysis performed, no single detection method is capable of detecting all threats in all air cargo commodity combinations. This thrust area is researching the use of multiple detection modalities through physics and mathematics based algorithms. This task is particularly difficult due to a lack of data from multiple detection modes for each threat/commodity combination. In FY08, an effort in data fusion was started at ORNL with a focus on both visualization and learning systems for data fusion. Under this initial effort, a framework for fusion was developed that would allow display of multiple imaging modalities together along with threat scores for an operator to evaluate. A publication was generated from this work (J.M. Beaver, R.A. Kerekes, and J.N. Treadwell. "Decision-level Information Fusion to Assess Threat Likelihood in Shipped Containers." Proceedings of the 2009 IEEE Conference on Technologies for Homeland Security, Waltham, MA, May 2009). With the change from a development project to a more R\&D project, the fusion effort shifted to INL for a physics based approach looking into combinations of multiple-energies and/or particles. A detailed technical report on this work is included with this report package under the filename Data Fusion for Air Cargo Explosive Screening v3.pdf.

\subsubsection{Non-Ionizing Radiation Thrust}

Based on expressed interest from potential customers, a short project was funded to determine the feasibility of including trace explosives sampling methods for enhanced detection of explosives in containerized/palletized cargo. As part of this effort, INL conducted a series of experiments during FY09 to measure the ability of various methods to remove various types of military explosives from surfaces relevant to air cargo. Removal/collection methods evaluated included mechanical methods (air flow and mechanical vibrations), thermal cycling, and combinations of thermal and mechanical methods. Explosives included TNT, Semtex and RDX fingerprints deposited on metal samples, on cardboard samples and on shrink-wrap samples. Results of these tests indicate that these three military explosives adhere strongly to metal surfaces and only an extremely small percentage of crystals could be removed by any of the methods. We were unable to remove any particles of RDX or SEMTEX from the metal surfaces using the combination methods. Removal of military explosives from cardboard and shrinkwrap surfaces using temperature/airflow and temperature/vibration were slightly more effective. 
Experimental data indicate that only the larger sized particles were removed by the removal methods evaluated. This information should be useful for designing filters and pre-concentrators for a trace explosives detection system for air cargo screening applications. The detailed technical report for this thrust effort is located in ACS_Trace_Report_DRAFT_1_Mar_10.doc.

Unintended emissions of electronic signals from trigger devices has been proposed as a potential method for detection of IEDs in air cargo. This method might be particularly effective in cargo commodities that should have no electronic components (e.g., fresh flowers or seafood). In a small research project conducted in FY08, it was found that cell-phones or two-way radios emit detectable frequencies even when the device is quiescent. This work is reported in Unintended Electronic Emissions (PNNL-19548).docx.

\subsubsection{High-Risk/High-Return Investments}

While the goal of the air-cargo initiative was to develop near-term screening solutions, it was recognized that a complete research portfolio should include some longer-term propositions for potential techniques still in their infancy, techniques that could have high impact if proven to be feasible. Two projects were initiated: an investigation of scatter techniques for better material identification (improvements to the "x-ray backscatter" technique) and a photon-tagging concept. Both of these projects were of the simulation/calculation flavor. The scatter study investigated a number of potential techniques, and found, in general, that for the air-cargo problem, the count rate and penetration considerations rendered these approaches unattractive. A complete discussion of this work can be found in X-Ray Scatter Variations (PNNL-19477).docx and XRD and Air Cargo (PNNL-19530).docx. The photon-tagging work asked if a device from the high-energy physics world could be practically modified to the MeV energies sufficient for air-cargo penetration, and if so, how could this device be used for explosives detection. A statistical analysis of detectability of explosive material in thin slabs was very promising, and simulations of the photon tagger showed promise as well. This work is reported on in Photon Tagging (PNNL-19549).docx. Follow-on funding to further explore the photon tagger has been obtained from NA-22 (project 3 in section 1.2.1).

\subsubsection{Project Planning}

As a result of a meeting with DHS S\&T in January of 2009, the team put together a project execution plan to outline the type of effort we felt was needed to develop a screening system for air cargo. This plan is based on a cyclic development that involves feedback from DHS, research groups, and suppliers throughout the cycle. The program execution plan is included in this documentation package under the file name "Battelle 2009 Air Cargo PEP 3-17.docx".

\section{Conclusions}

Air cargo screening is a difficult challenge that will require significant investment in both research and development to find a suitable solution to ensure the safety of passengers without significantly hindering the flow of commodities. The initiative funded by Battelle has positioned this group to make major contributions in meeting the air cargo challenge by developing collaborations, developing laboratory test systems, improving knowledge of the challenges (both technical and business) for air cargo screening, and increasing the understanding of the capabilities for current inspection methods ( $x$ ray radiography, $x$-ray backscatter, etc.) and potential future inspection methods (neutron radiography, fusion of detector modalities, advanced trace detection, etc.).

Lastly, air cargo screening is still an issue that will benefit from collaboration between Department of Energy Laboratories and Battelle. On January 7, 2010, DHS Secretary Napolitano joined White House 
Press Secretary Robert Gibbs and Assistant to the President for Counterterrorism and Homeland Security John Brennan to announce several recommendations DHS has made to the President for improving the technology and procedures used to protect air travel from acts of terrorism. (This announcement followed the 25 Dec'09 Delta / Northwest Airlines Flight 253 terror attack.) Secretary Napolitano outlined five recommendations DHS will pursue to enhance the safety of the traveling public. One of the five recommendations, read as follows: "Establish a partnership on aviation security between DHS and the Department of Energy and its National Laboratories in order to develop new and more effective technologies to deter and disrupt known threats and proactively anticipate and protect against new ways by which terrorists could seek to board an aircraft." In conclusion, it appears very timely that Battelle and its DOE lab partners initiated a serious collaboration on the air cargo topic, and that we should continue to work toward future collaboration in response to the government's needs. 